\title{
Effects of ultra-micro powder wuji wan on gut microbes and enzyme activities
}

\author{
ZhouJin Tan ${ }^{1 *}$, Ao Zeng ${ }^{1}$, XinHua Shu², NenQun Xiao ${ }^{1}$, HuaLing Zhang ${ }^{1}$, KangXiao Guo ${ }^{1}$ and GuangXian Cai ${ }^{1 *}$ \\ *Correspondence: tanzhjin@yahoo.com.cn and tanzhjin@sohu.com \\ ${ }^{1}$ Hunan University of Traditional Chinese Medicine, Changsha, Hunan Province, 410208, PRC. \\ ${ }^{2}$ Department of Life Sciences, Glasgow Caledonian University, Glasgow G4 OBA, United Kingdom.
}

\begin{abstract}
To explore the effect of Chinese medicine ultra-micro powder Wuji Wan on gut microorganisms and enzyme activities, microbial culturing methods and enzymological methods were used to determine the amount of microbes and enzyme activities in gastrointestinal tract. Antibacterial activity of ultra-micro powder Wuji Wan were determined in vitro. Mice were randomly divided into 6 groups. The drugs were orally administered. The results showed that the amount of Bifidobacterium spp., Lactobacillus spp., and the activity of amylase and cellulase in gastrointestinal tract were not significantly affected by ultra-micro powder Wuji Wan $(\mathrm{P}>0.05)$. But the growth of bacteria, colibacillus and fungi, and the activity of the protease and xylanase in gastrointestinal tract were inhibited by the ultra-micro powder Wuji Wan $(\mathrm{P}<0.05)$. Ultra-micro Wuji Wan can significantly inhibit the growth of common opportunistic pathogens in gastrointestinal tract in vitro. The effects of ultra-micro powder Wuji Wan on microorganisms and enzyme activities in stomach were stronger than in intestine. The $25 \%$ of ultra-micro powder dose can produce the greatest effect in the stomach without injection. Ultra-micro technology can conserve Chinese herbal medicine.
\end{abstract}

Keywords: Wuji Wan, ultra-micro powder Wuji Wan, gut microbes, gut enzyme

\section{Introduction}

The Traditional Chinese Medicine (TCM) ultra-micro powder technology is the rising high-tech in recent years. The applications of the ultra-micro powder Chinese herbal pieces had a very important practical significance and value on both improving the effect and quality of Traditional Chinese Medicine and reducing the waste of Traditional Chinese Medicine resources. It's very useful for the modernization and internationalization of Chinese medicine [1]. But the appropriate clinical dosage of ultra-micro powder of TCM needed for the treatment is still not clear [2].

There are about $10^{14}$ microorganisms in human gastrointestinal tract, a huge micro-ecological system [3-4]. It is important to parasis for a variety of physiological function (especially metabolic) as well as life activities for gastrointestinal flora [5]. At the same time, a number of investigations have shown that certain butyrate-producing firmicute bacteria are reduced in inflammatory bowel disease [6]. The human gastrointestinal microbes and enzymes dissolve and transform the Chinese medicines through hydrolysis and reduction reactions [7]. The metabolic function of intestinal microbes may play important roles on the effect of Chinese medicine on diseases [8-10].
Wuji Wan, a prescription of TCM, consists of three herbs: Rhizoma Coptidis, Fructus Evodiae Rutaecarpae and Radix Paeoniae Alba. It has excellent curative effects on gastrointestinal diseases [11]. Wuji Wan have been used to treat stomach pain, Tunsuan, abdominal pain and diarrhea due to incoordination between the liver and the spleen [12], and in particular the treatment of gastric ulcer. Like all other Chinese medicine, the curative mechanisms of Wuji Wan are not fully understood [13]. Previous studies showed that coptis, fructus evodiae and peony from Wuji Wan had apparent antibacterial effects [14-15]. Ultra-micro powder technology has been used for Chinese medicine compound preparation, this can increase the contents and the dissolution of effective ingredients. The ultra-micro powder Wuji Wan contain higher levels of berberine hydrochloride, paeoniflorin, evodianmine and rutaecarpine compared with traditional Wuji Wan. In this study, we aimed with investigate the impacts of ultra-micro powder Wuji Wan on gut microbes and enzyme activities in mice.

\section{Materials and methods \\ Experimental animals}

60 Kunming mice (SPF grade) were provided by Shanghai Experimental Animal Center of Chinese academy of Sciences. 
The weight of each mouse was about $20 \pm 2 \mathrm{~g}$. Mouse food was provided by Experimental Animal Center of Hunan University of Traditional Chinese Medicine.

\section{Drug treatment}

Wuji Wan were prepared according to the Chinese Pharmacopoeia 2010. 300g of Rhizoma coptidis, $50 \mathrm{~g}$ of Fructus evodiae and $300 \mathrm{~g}$ of Radix paeoniae alba were ground into a fine powder. The ultra-micro powder Wuji Wan were made by Ultra-powder Chinese Medicine Engineering Research Center of Hunan Academy of Chinese Medicine. The Wuji Wan water decoction and the ultra-micro powder Wuji Wan water decoction( $100 \%$ dose) were made into water decoction without filter and centrifuge, and the concentration of both water decoction were $0.069 \mathrm{~g} / \mathrm{ml}$ [16]. The Ultra-micro powder Wuji Wan water decoction was diluted to $50 \%, 25 \%$ and $12.5 \%$ dose. Mice were randomly divided into 6 groups (10 mice per group): normal control animals, Wuji Wan treated animals, ultra-micro Wuji Wan treated animals, $50 \%$ of ultra-micro Wuji Wan treated animals, $25 \%$ of ultra-micro Wuji Wan treated animals, and $12.5 \%$ of ultra-micro Wuji Wan treated animals. All the drugs were orally administered to the mice by gavage twice a day $(0.3 \mathrm{ml}$ each time) [16]. The normal control mice were treated with boiled water.

\section{Media}

For bacteria: Beef extract-peptone medium [17], for colibacillus: EMB medium [17], for Lactobacillus spp.: MRS medium [18], for Bifidobacteria spp.: BBL medium [19], for fungi: martin Rose Bengal Medium [17].

\section{Microbial strains}

Standard microbial strains: Candida albican, Staphyloccocus aureus, Salmonella sp., Escherichia coli and Pseudornonas aeruginosa were provided by Microbiology Teaching and Research Section of Hunan University of Traditional Chinese Medicine.

\section{Extraction of gastrointestinal contents}

All the mice were sacrificed on the morning of the ninth day after treatment. Mouse intestinal (jejunum to the rectum) and gastric contents were collected in a steriled environment.

\section{Determination of microorganisms from gastrointestinal contents}

A certain amount of intestinal and gastric contents were weighed in a steriled environment and transferred into conical flask equipped with glass beads and steriled water. In order to release microorganisms from intestinal and gastric contents into steriled water completely, the conical flasks were put on a table concentrator for $30 \mathrm{~min}$ with 120 rpm shaking. The number of microorganisms of intestinal and gastric contents was determined with the method of plate culture counting. Total numbers of bacteria and colibacillus were counted after culturing 24 hours at $37^{\circ} \mathrm{C}$. Total numbers of Lactobacillus spp., Bifidobacteria spp. and fungi were determined after culturing 48 hours at $37^{\circ} \mathrm{C}$.

\section{Analyze enzyme activities of mice stomach and intestinal contents}

To dissolve enzyme from stomach and intestinal contents completely, the contents were diluted with steriled water and heated preservation for $30 \mathrm{~min}$ in a $40^{\circ} \mathrm{C}$ water bath. The enzyme extracts were centrifuged for $10 \mathrm{~min}$ at $2000 \mathrm{rpm}$, following which the enzyme activities in the supernatant were analyzed. The activities of amylase, xylase and cellulase activities were determined by DNS colorimeter as previous described [20]. Cellulase activity was defined as a unit of the cellulase activity as generating $1 \mathrm{mg}$ reducing sugar of $1 \mathrm{~g}$ contents at $46^{\circ} \mathrm{C}$ for $30 \mathrm{~min}$. Amylase activity was defined as a unit of the amylase activity as generating $1 \mathrm{mg}$ reducing sugar of $1 \mathrm{~g}$ contents at $37^{\circ} \mathrm{C}$ for $60 \mathrm{~min}$. Xylase activity was defined as a unit of the xylase activity as generating $1 \mathrm{mg}$ reducing sugar of $1 \mathrm{~g}$ contents at $46^{\circ} \mathrm{C}$ for $60 \mathrm{~min}$. Protease activity was determined by Folinphend method [21]. Protease activity was defined as a unit of protease activity as generating $1 \mathrm{~g}$ amino acid of $1 \mathrm{~g}$ contents at $37^{\circ} \mathrm{C}$ for $40 \mathrm{~min}$.

The measurement of the antimicrobial cycle dimension The antibacterial activities of Wuji Wan and ultra-micro Wuji Wan were tested using agar slant culturing medium. The Wuji Wan water decoction and the ultra-micro powder Wuji Wan water decoction were enriched to $1 \mathrm{~g} / \mathrm{ml}$ by filtering and centrifuging. Microbe suspension was made with a mixture of tested strains and steriled water at $10^{6}-10^{7} \mathrm{CFU} /$ $\mathrm{ml}$ and applied onto plate culture. After static cool-down

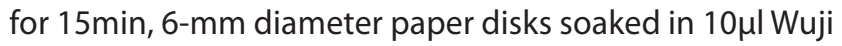
Wan water decoction or ultra-micro powder Wuji Wan decoction were placed on the inoculums. Disks soaked in $10 \mu \mathrm{l}$ of steriled water were used as negative controls. The dimension of growth inhibition was measured using a calibrated ruler after the antimicrobial cycle was formed. The experiments were repeated three times.

\section{Statistical analysis}

Measurement data of every group were represented with mean-standard deviation $(\bar{X} \pm s)$ and analyzed using DPS v7.05 statistical software.

\section{Results}

\section{Effect of Wuji Wan and ultra-micro Wuji Wan on} treated mice

When compared with normal control mice treated with boiled water, all mice treated with Wuji Wan or ultra-micro Wuji Wan did not show any sign of sluggishness, aversion to cold, or diarrhea, and did not reduce food intake. The weight of mice in each treated group was similar to that of 


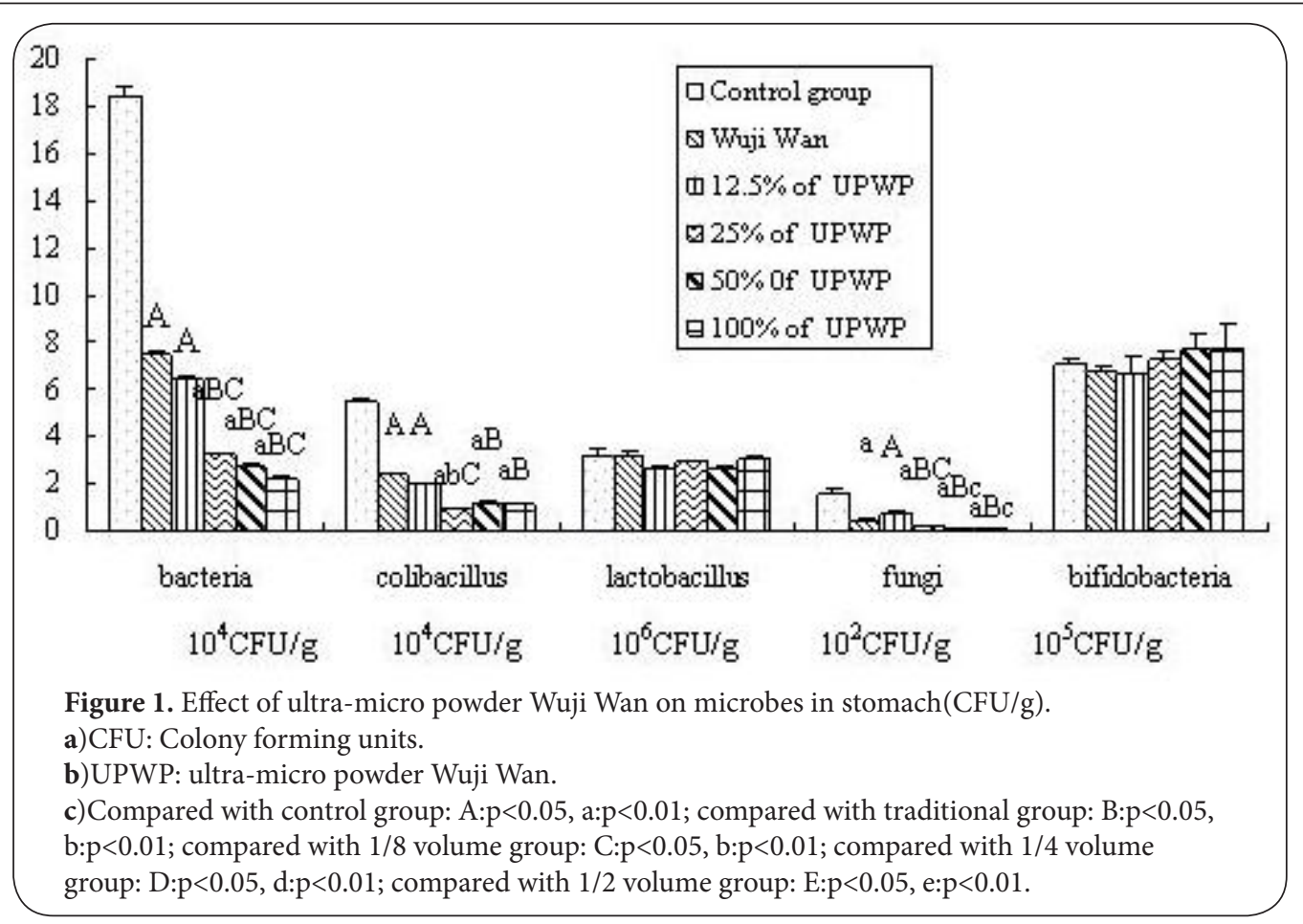

control mice. It is suggested that Wuji Wan and Ultra-micro Wuji Wan had no effect on physiological activity in mice.

\section{Effect of ultra-micro powder of Wuji Wan on on mice stomach microbes}

The amount of flora in stomach is relatively very low because of the lower pH. The Lactobacillus spp., Bifidobacteria spp. and Streptococcus faecalis are beneficial bacteria in the gastrointestinal tract, other bacteria are generally opportunist pathogens and pathogenic.we could see from Figure 1, the amount of Lactobacillus spp. and Bifidobacteria spp. in stomach were significantly higher than the amount of other opportunist pathogens, but Lactobacillus spp. and Bifidobacteria spp. of each Chinese medicine treated group showed no significant difference $(P>0.05)$, suggesting both Wuji Wan and ultra-micro powder Wuji Wan had no effects on the growth of Lactobacillus spp and Bifidobacteria spp. in stomach. The amount of bacteria, colibacillus and fungi in mice treated with Wuji Wan or $12.5 \%$ of ultra-micro powder Wuji Wan were significant lower than those in control group's mice $(P<0.05)$. However, there was no significant difference between $12.5 \%$ of ultra-micro powder Wuji Wan and Wuji Wan group ( $P>0.05)$, suggesting $12.5 \%$ of ultramicro powder Wuji Wan has a similar effect to the Wuji Wan. After treatment with $25 \%$ of ultra-micro powder Wuji Wan, $50 \%$ of ultra-micro powder Wuji Wan and whole ultra-micro powder Wuji Wan the amount of bacteria, colibacillus and fungi in the treated groups declined significantly $(P<0.01)$. Compared with $12.5 \%$ of ultra-micro powder Wuji Wan and traditional Wuji Wan group, the amount of bacteria and fungi in $25 \%, 50 \%$ and whole ultra-micro powder Wuji
Wan group declined significantly $(\mathrm{P}<0.05)$. Compared with traditional Wuji Wan group, the amount of colibacillus in $25 \%, 50 \%$ and whole ultra-micro powder Wuji Wan groups also declined significantly $(P<0.05)$ (Figure 1$)$. The results suggest that Wuji Wan and ultra-micro powder Wuji Wan can inhibit the growth of opportunist pathogens and pathogenic bacteria; the inhibiting effect of $25 \%, 50 \%$, and whole ultra-micro Wuji Wan were superior to $12.5 \%$ of ultra-micro powder Wuji Wan and traditional Wuji Wan.

\section{Effect of ultra-micro powder Wuji Wan on mice stomach enzyme activities}

Gut enzymes come from dietary intake and secretion by gastrointestinal cells and microbial cells. Cellulase and xylase are secreted only by gastrointestinal microbes, not by the mouse itself. So the change of cellulase and xylase activities could reflect the change of microbes that secrete cellulase and xylase. We measured stomach enzyme activities in control and drug treated mice, cellulase activities were not significantly different in medincine treatment and control group $(P>0.05)$. There was a remarked difference of xylase activities between Chinese medicine treatment group and control group $(P<0.05)$, Xylase activities in groups treated with $25 \%, 50 \%$, and whole ultra-micro powder Wuji Wan reduced more $(\mathrm{P}<0.05)$ (Figure 2). Amylase and protease are mainly secreted by the mouse itself. Amylase activities of all Chinese medicine treated groups showed no significant change compared with that of control group $(P>0.05)$. However, the protease activities of all Chinese medicine treated group significantly reduced $(P<0.05)$ (Figure 2). The protease activities of $25 \%, 50 \%$ and whole 


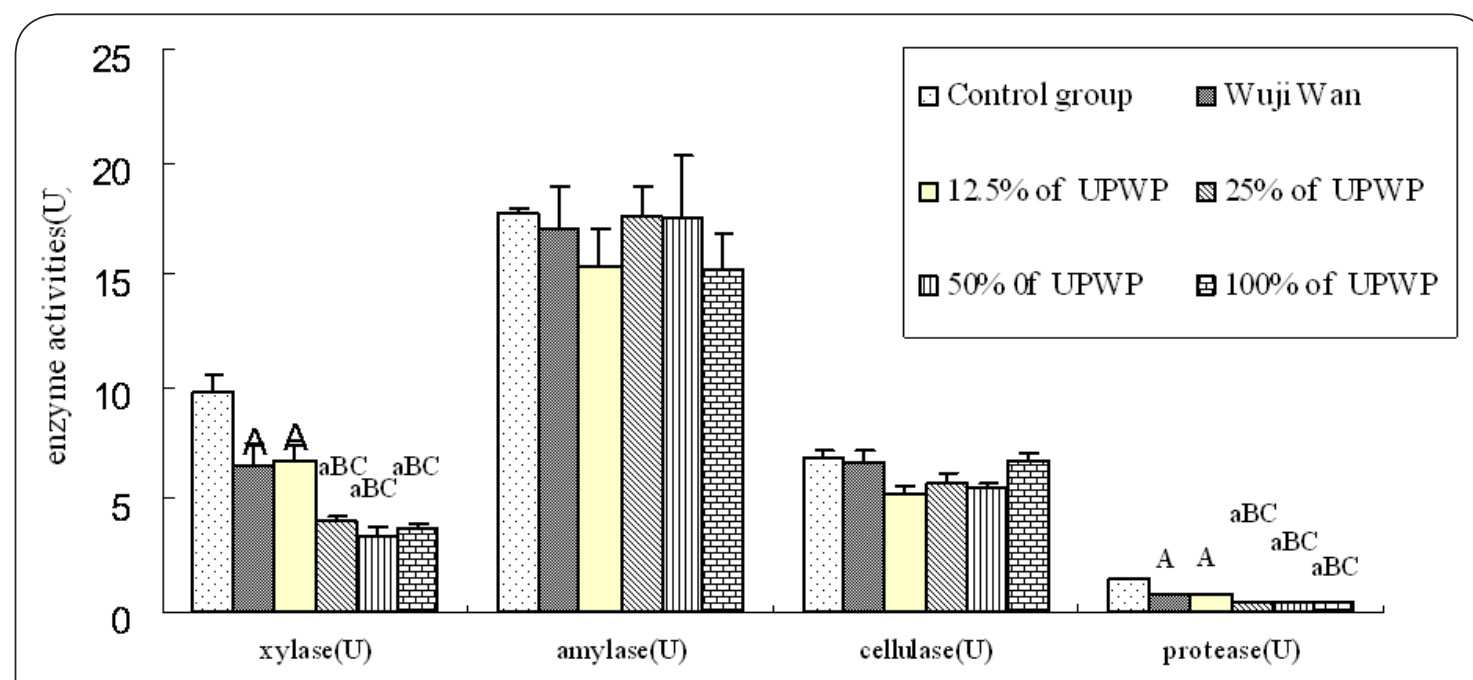

Figure 2. Effect of ultra-micro powder Wuji Wan on stomach enzyme activities.

The activities of xylase, amylase, cellulase and protease were measures as described in Materials and Methods. a)Compared with control group: A:p $<0.05$, a:p $<0.01$; compared with traditional Wuji Wan treated group: $\mathrm{B}: \mathrm{p}<0.05$; compared with $12.5 \%$ of ultra-micro powder Wuji Wan treated group: C:p $<0.05$. b)UPWP: ultra-micro powder Wuji Wan.

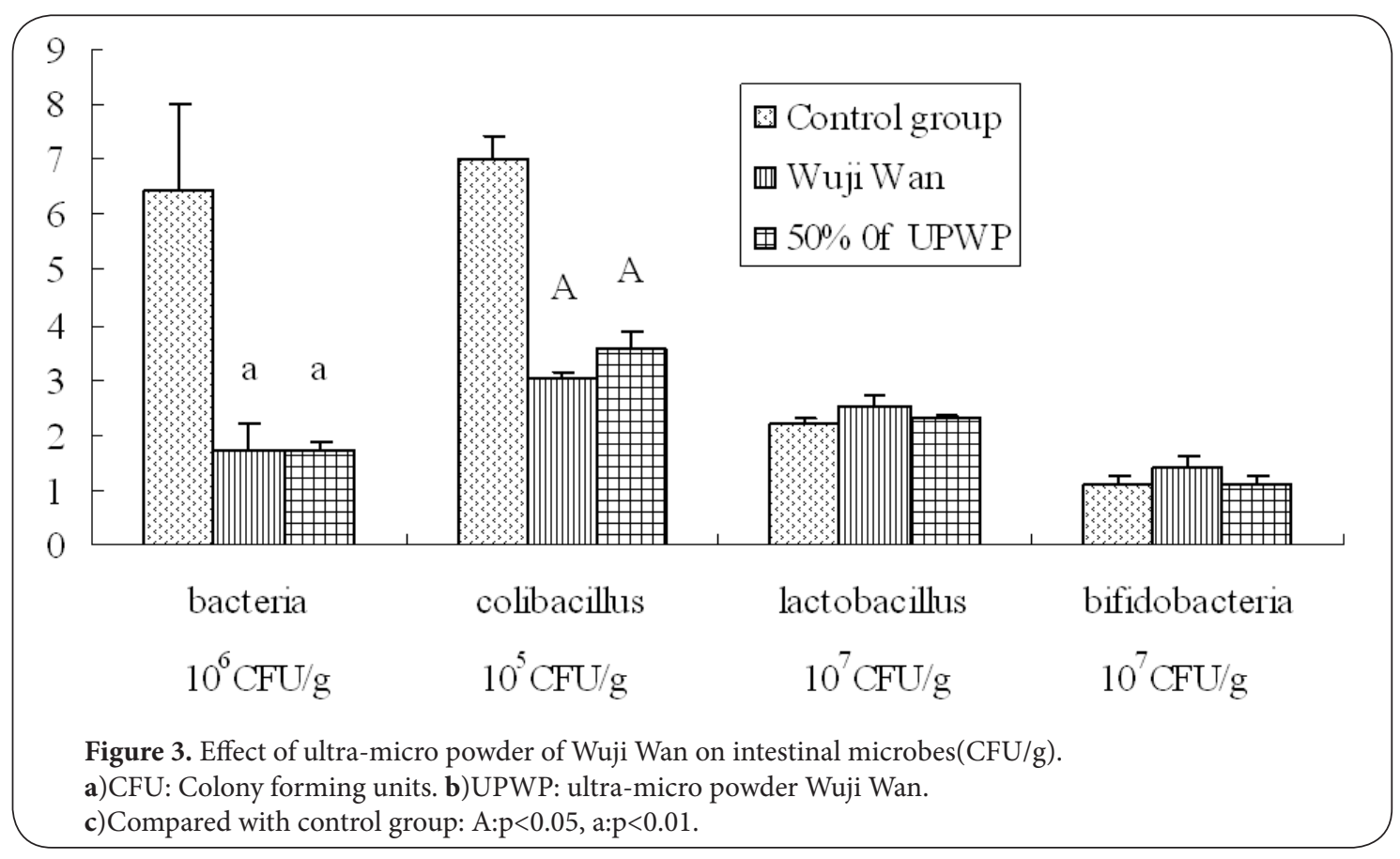

ultra-micro powder Wuji Wan group were significant reduced compared with $12.5 \%$ ultra-micro powder Wuji Wan and traditional Wuji Wan group $(\mathrm{P}<0.05)$.

\section{Effect of ultra-micro powder Wuji Wan on mice intestinal} microbes

In our previous study $50 \%$ of ultra-micro powder Chinese medicine on intestinal microbes and enzyme activities was the best [22], so we adopted $50 \%$ of ultra-micro powder Wuji Wan as a working dose to investigate its effect on intestinal microbes and enzyme activities. The amount of intestinal microbes were significantly higher than in stomach because of elevated $\mathrm{pH}$ value (Figure 3 ). The amount of Lactobacillus spp. and Bifidobacteria spp. of Chinese medicine treated group were similar to the control group ( $P>0.05)$. Compared with control group, the amount of bacteria and colibacillus in 50\% ultramicro powder Wuji Wan and traditional Wuji Wan group 


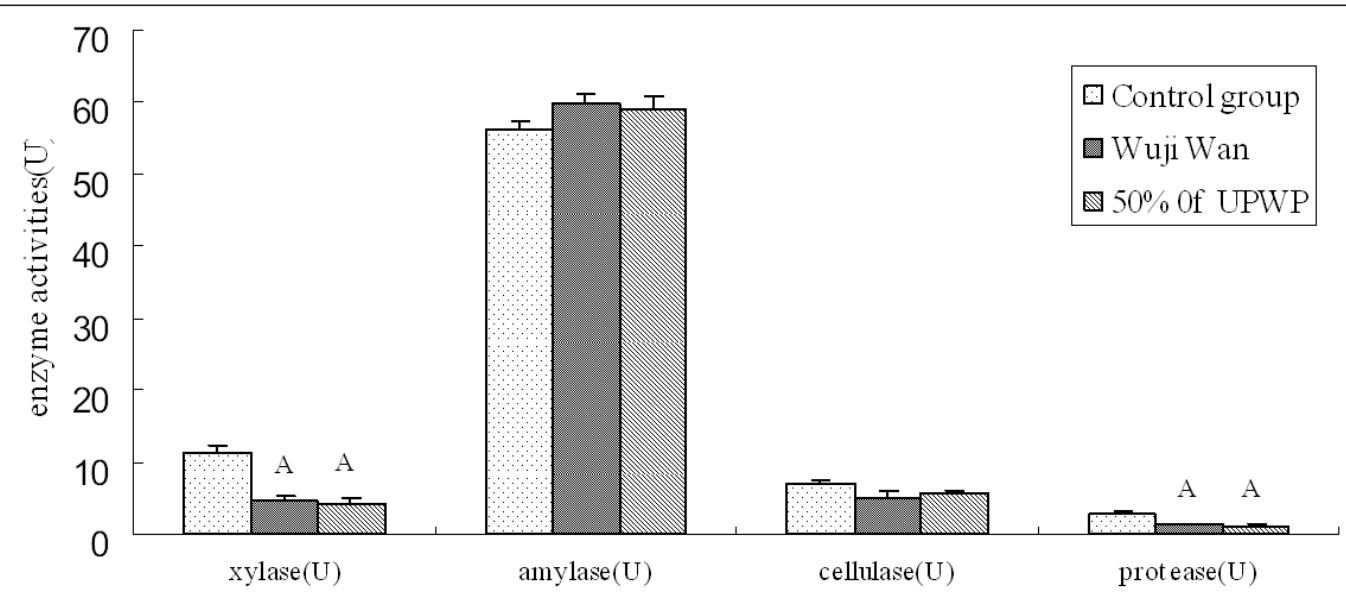

Figure 4. Effect of ultra-micro powder Wuji Wan on intestinal enzyme activities in mice(U).

The activities of xylase, amylase, cellulase and protease were measures as described in Materials and Methods.

a) Compared with control group: A:p $<0.05$.

b) UPWP: ultra-micro powder Wuji Wan.

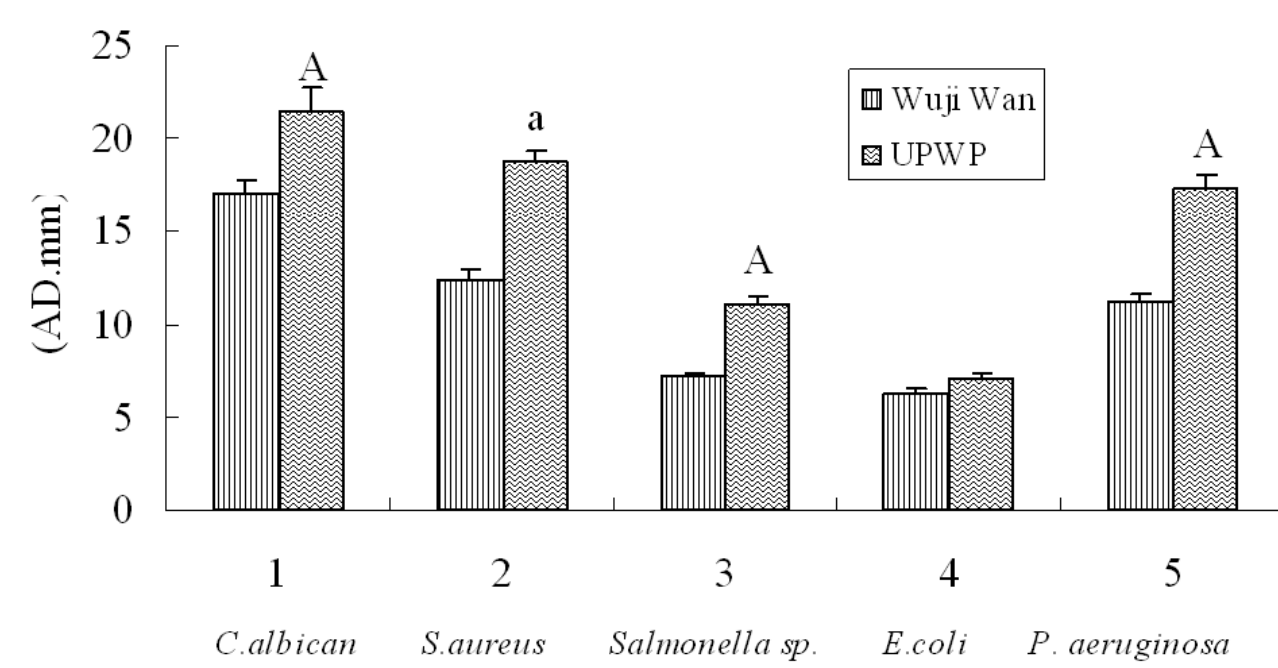

Figure 5. The anti-microbial test of Wuji Wan and ultramicro powder Wuji Wan in vitro(AD.mm). a) $\mathrm{AD}$ : Inhibition zone diameter.

b)Compared with traditional decoction group: A:p $<0.05$, a:p $<0.01$.

c) UPWP: ultra-micro powder Wuji Wan.

significantly declined (Figure 3 ). There is no significant difference between $50 \%$ ultra-micro powder Wuji Wan and traditional Wuji Wan group $(P>0.05)$.

\section{Effect of ultra-micro powder Wuji Wan on intestinal enzyme activities in mice}

We could see from the Figure 2,4. The activities of intestinal amylase was 3.5 times higher than amylase in stomach, while the activity of intestinal protease was two times higher than protease in stomach $(\mathrm{P}<0.01)$ (Figure 2,4). It is possible that the small intestine is the major organ for digestion and absorption. When compared with the control group, amylase activity in the $50 \%$ of ultra-micro powder
Wuji Wan and traditional Wuji Wan group both slightly increased. Cellulase activity of both group slightly decreased (Figure 4), but there were no significant difference ( $>0.05$ ). The xylase and protease activities of Chinese medicine treated group significantly decreased compared with the control group $(\mathrm{P}<0.05)$ (Figure 4).

\section{The anti-microbial test of Wuji Wan and ultramicro} powder Wuji Wan in vitro

Candida albican, Staphyloccocus aureus, Salmonella sp., Escherichia coli and Pseudornonas aeruginosa are opportunistic pathogens and pathogenic bacteria in the grastrointesetinal tract. Four bacterial species 
(S. aureus, Salmonella sp., E. coli and P. aeruginosa) used in the experiments were grown on beef extract-peptone medium, fungi $C$. albican was grown on martin rose bengal medium. Figure 5 shows that both traditional Wuji Wan and ultra-micro powder Wuji Wan could inhibit the growth of all bacteria and fungi, with particular effectiveness for C. albican, S. aureus, Salmonella sp. and P. aeruginosa. The inhibition zones of ultra-micro powder Wuji Wan were significant bigger than the traditional Wuji Wan, suggesting ultra-micro powder Wuji Wan has higher effectiveness to inhibit growth of microbes.

\section{Discussion}

Under the circumstances of increasing antibiotic resistance in bacteria and antibiotic adverse reactions, a detailed study and accurate understanding of the function of natural plant ingredients will enable us to develop Chinese medicine as novel antimicrobial drugs. The present study showed that Wuji Wan and ultra-micro powder Wuji Wan can inhibit the growth of opportunistic pathogens and pathogenic bacteria in the grastrointesetinal tracts but have no effect on probiotics. There are at least six bioactive alkaloids: berberine, coptisine, jatrorrhizine, palmatine, evodiamine and rutacarpine identified in Wuji Wan [23]. most of the alkaloids have antibacterial activities (such as anti H.pylori and E. coli) [24-25]. Pepsin has been considered to be the attack factor for stomach diseases (such as gastritis, gastric ulcer and gastric cancer), our results showed that both traditional Wuji Wan and ultramicro powder Wuji Wan can restrain xylase activities. It is possible that berberine embeds pepsin and trypsin molecules through hydrophobic interaction to form a static quenching caused by a particular structure of the association complex, and can function for a long time in the gastrointestinal tract because of the relatively poor fat solubility [26]. The mechanism for the treatment of gastrointestinal diseases is that Wuji Wan can inhibit the growth of opportunist pathogens and pathogenic bacteria, and can inhibit protease activity in stomach.

Wuji Wan are the classical recipe for the clinical treatment of peptic ulcer and gastritis, but the residence time of traditional Wuji Wan in the stomach is very short, usually for about two hours. It is difficult to form the effective concentration in the stomach. However, ultra-micro powder Wuji Wan could solve this problem as the bioactive ingredients of wall-breaking ultra-micro powder Wuji Wan can be rapidly dissolved in the stomach where it could form the highest concentrations of its active ingredients. But in terms of traditional Chinese medicine, the adhesion to intestinal walls is poor and traditional Wuji Wan still contain intact cell walls. When traditional Chinese medicine are orally administrated, the bioactive ingredients are released through wall-breaking by microbes and enzymes in the gastrointestinal tract [7]. Ultra-micro Chinese medicine has the surface, volume, quantum and macro tunnel effects which can strengthen the adhesion to the intestinal walls [27-28]. Our results showed the ultra-micro powder Wuji Wan are more effective than traditional Wuji Wan in inhibiting the growth of bacteria, colibacillus and fungi. Previous studies have shown that fungi and bacillus can excrete xylase and cellulase [29-31]. The xylase activities of ultra-micro powder Wuji Wan group significantly decreased in stomach, but the cellulose activities only slightly decreased, suggesting there are possibly other unknown reason causing for the decline of enzyme activities. Both traditional Wuji Wan and ultra-micro powder Wuji Wan also could inhibit the growth of intestinal bacteria and colibacillus, and lead to the decline of xylase and protease activities. Overall our study suggests ultra-micro powder Wuji Wan are more effective than traditional Wuji Wan in modulating the gut microbial system, and provides insights for understanding the mechanisms of Wuji Wan in treating gastrointestinal diseases.

\section{Competing interests}

The authors declare that they have no competing interests.

\section{Authors' contributions}

ZhouJin Tan conceived of the study, and participated in its design and coordination and helped to draft and revise the manuscript. Ao Zeng participated in its design and acquisition of data and helped to draft and revise the manuscript. XinHua Shu participated in its interpretation of data and helped to draft and revise the manuscript. NenQun Xiao made substantial contributions to analysis and interpretation of data. HuaLing Zhang made substantial contributions to interpretation of data. KangXiao Guo participated in the detection of enzyme activies. GuangXian Cai made substantial contributions to conception and design and coordination. All authors read and approved the final manuscript. There won't be any further changes in the authorship which includes either addition or removal of author's details and ZhouJin Tan will be sole responsible person for all the communications and proceedings that are needed to be done with the publisher (According to the necessity of the publisher) on behalf of all the authors.

\section{Acknowledgement and funding}

We are grateful to the financial support of the National Natural Science Foundation of China (81173214) and the Key Project of Hunan Science and Technology Agency (2010SK2002).

\section{Publication history}

Received: 01-Dec-2012 Revised: 23-Jan-2013

Re-Revised: 30-Jan-2013 Accepted: 05-Feb-2013

Published: 20-Feb-2013

\section{References}

1. Li Y, Cai G X and Peng S: Comparative Study on Pharmacological Actions of Ultra-quick-lysotype Xiao Caihu Decoction and Traditional Decoction. Chinese Journal of Traditional Medical Science and Technology 2007,14:343-344. (in Chinese).

2. Cai G X,Yang Y H and Li Y H: Ultra-micro powder Technology and Reform in Preparation of Pieces of Medicinal Herbs in Traditional Chinese Medicine. World Science and Technolog-modernization of Tradtional Chinese Medcine and Materia Medicine 2004, 6:67-70. (in Chinese).

3. Backhed F: Addressing the gut microbiome and implications for 
Tan et al. Journal of Pharmaceutical Technology \& Drug Research 2013, http://www.hoajonline.com/journals/pdf/2050-120X-2-9.pdf

doi: $10.7243 / 2050-120 \mathrm{X}-2-9$

obesity. Int. Dairy. J 2010, 20:259-261. | Article

4. Paliy $\mathrm{O}$, Kenche $\mathrm{H}$, Abernathy $\mathrm{F}$ and Michail S: High-throughput quantitative analysis of the human intestinal microbiota with a phylogenetic microarray. Appl Environ Microbiol 2009, 75:3572-9. | Article | PubMed Abstract | PubMed Full Text

5. Louis $\mathrm{P}$ and Flint $\mathrm{H} \mathrm{J}$ : Diversity, metabolism and microbial ecology of butyrate-producing bacteria from the human large intestine. FEMS Microbiol Lett 2009, 294:1-8. | Article | PubMed

6. Sokol H, Lepage P, Seksik P, Dore J and Marteau P: Molecular comparison of dominant microbiota associated with injured versus healthy mucosa in ulcerative colitis. Gut 2007, 56:152-4. | Article | PubMed Abstract | PubMed Full Text

7. Vitali B, Ndagijimana M, Cruciani F, Carnevali P, Candela M, Guerzoni $\mathrm{M} E$ and Brigidi $P$ : Impact of a synbiotic food on the gut microbial ecology and metabolic profiles. BMC Microbiol 2010, 10:4. | Article | PubMed Abstract | PubMed Full Text

8. Bae E A, Shin J E and Kim D H: Metabolism of ginsenoside Re by human intestinal microflora and its estrogenic effect. Biol Pharm Bull 2005, 28:1903-8. | Article | PubMed

9. Blaut $\mathrm{M}$ and Clavel $\mathrm{T}$ : Metabolic diversity of the intestinal microbiota: implications for health and disease. J Nutr 2007, 137:751S-5S. | Article | PubMed

10. Sekirov I, Russell S L, Antunes L C and Finlay B B: Gut microbiota in health and disease. Physiol Rev 2010, 90:859-904. I Article I PubMed

11. Tan H, Liu B Y, Cai Y and Cai G X.: Study on the clinical efficiency and inflammatory mechanism of ultra-podwer Wuji Wan on patients with gastric ulcer in syndrome of stomach and liver stagnation-hot. Chinese Journal of Integrated Traditional And Western Medicine on Digestion 2010, 18,10. (in Chinese).

12. Liu W, Si N,Chen L F, Feng G and Yang S: Advancement of Wuji Wan. Journal of Guiyang College of TCM 2011,33:86-87. (in Chinese).

13. Zhang $S$ and Yang J: Progress in gastrointestinal micro-ecology in integration of traditional Chinese medicine and Western medicine. World Chinese Journal of Digestology 2008,161:3135-3138. (in Chinese).

14. Gong H M, Wang Z J and Duan W Z: Study on the Analgesia, anti-inflammatory effects of WujiWan. Chinese Journal of Traditional Medical Science and Technology 1998, 5:147-148. (in Chinese).

15. Gong $M X$,Wang Z M,Zhang Q W, Li J R, Song Y F,Zhao $H$ and Chen Y H: Advancement of Pharmacological Research on the Effective Components in Fructus Evodiae. Traditional Chinese Drug Research \& Clinical Pharmacology 2009,20:183-186. (in Chinese).

16. He SL, Wang J, Wang JJ: Traditional Chinese medicine scientific research design and statistics. Changsha: Hunan Science and Technology Press 2006. (in Chinese).

17. Shen $\mathrm{P}$ and Chen X D: Microbiology experiment. Peking:Higher education press 2008, (in Chinese).

18. Ling D W: Actic acid bacteria classification and identification and experimental method. Peking: China Light Industry Press 1999. (in Chinese).

19. Zhang C W: Sanitary microbiology. Peking:People's Medical Publishing House 2007. (in Chinese).

20. Zhu J,Cao K M and Zhou R Q: Biochemistry Experiment. Shanghai:Shanghai Science and Technology Press1981. (in Chinese).

21. Su S Y, Jiang Y J and Shen Y: Research in correlation between Shanghai brewing Aspergillus oryzae proteinase and the amount of spores. China Condiment 2010,5:48-52. (in Chinese).

22. Tan Z J,Wu H, Liu F L, Cai Y, Cai G X,Zhang H L and Zeng A: Effect of ultra-micro powder qiweibaishusan on the intestinal microbiota and enzyme activities in mice.Acta Ecological Sinica 2012, 32(21):68566863. (in Chinese).

23. Yuan J, Wang Y, An R, Wang S, Li S J, Jia J Y, Bligh S W, Wang X H and Ma Y M: Simultaneous determination of six alkaloids and one monoterpene in rat plasma by liquid chromatography-tandem mass spectrometry and pharmacokinetic study after oral administration of a Chinese medicine Wuji Pill. J Chromatogr B Analyt Technol Biomed
Life Sci 2012, 895-896:154-61. | Article | PubMed

24. Pepeljnjak S and Petricic J: The antimicrobic effect of berberine and tinctura berberidis. Pharmazie 1992, 47:307-8. I PubMed

25. Yang $C M, M a Z P$ and Wang $Z P$ : Berberine,ethidium bromide,sodium dodecyl sulfate (SDS) on the elimination of the resistance plasmid of Shigella. Northwest Pharmaceutical Journal 2000,15:64-65. (in Chinese).

26. He K D, Leng X L, Li L, Tan J, Liu Z F and Liu K w: Study of the Interaction between Barberine and Trypsin. Natural Product Research and Development 2008,20:488-490. (in Chinese).

27. Laparra J M and Sanz Y: Interactions of gut microbiota with functional food components and nutraceuticals. Pharmacol Res 2010, 61:219-25. | Article | PubMed

28. Hao $\mathrm{Z} \mathrm{H}$ and Zhang B W: Superfine milling technique used for Chinese medicine materials processing. Chinese Journal of Veterinary Drug 2003,37:50-52. (in Chinese).

29. Aro N, Ilmen M, Saloheimo A and Penttila M: ACEl of Trichoderma reesei is a repressor of cellulase and xylanase expression. Appl Environ Microbiol 2003, 69:56-65. | Article | PubMed Abstract | PubMed Full Text

30. Shi Y,Jiang A Q and Lu L: Advanced in Microbiological Mechanism and Application of Straw Degradation. J. Microbiol 2002,22:47-50.

31. Hamasaki N, Ishii E, Tominaga K, Tezuka Y, Nagaoka T, Kadota S, Kuroki T and Yano I: Highly selective antibacterial activity of novel alkyl quinolone alkaloids from a Chinese herbal medicine, Gosyuyu (Wu-Chu-Yu), against Helicobacter pylori in vitro. Microbiol Immunol 2000, 44:9-15. | PubMed

\section{Citation:}

Tan Z, Zeng A, Shu X, Xiao N, Zhang H, Guo K and Cai G: Effects of ultra-micro powder wuji wan on gut microbes and enzyme activities. journal of Pharmaceutical Technology and Drug Research 2013, 2:9.

http://dx.doi.org/10.7243/2050-120X-2-9 